\title{
Individual deviation from behavioural correlations: a simple approach to study the evolution of behavioural syndromes
}

\author{
Gábor Herczeg \& László Zsolt Garamszegi
}

\begin{abstract}
The study of correlations between different behaviours in a population-referred to as behavioural syndromes-has begun to flourish during recent years. However, the evolutionary mechanisms that cause behavioural traits to vary non-independently from each other are still poorly understood. Here, we bring behavioural syndromes into a new perspective, in which the phenomenon is regarded at the individual level and on a continuous scale instead of as a population-level presence/absence trait. As the correlation between behaviours is never perfect (i.e. $r<$ 1), individuals are likely to vary in how consistently they behave. Therefore, we can predict that if behavioural syndromes at the population level are results of natural selection, the consistency in a suite of behaviours-and not the behavioural configuration per se-should be heritable and involve fitness advantages at the individual level. We define a variable that describes the individual deviation
\end{abstract}

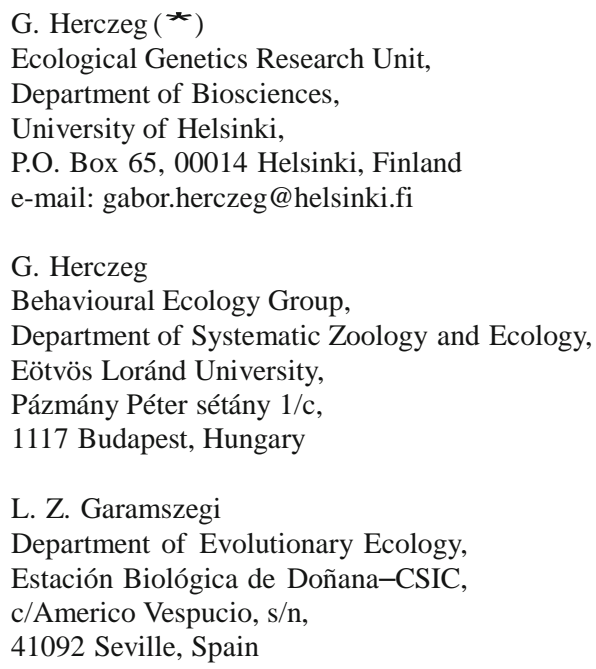

from the hypothetical perfect correlation predicted by the syndrome. The use of such a variable depicting the consistency of behaviours of individuals allows us to make solid evolutionary inferences about correlated behaviours from patterns of individual instead of population variation. We suggest that, by adopting the concept of syndrome deviation, understanding the evolution of behavioural syndromes and, in particular, testing competing evolutionary hypotheses about the origin of behavioural syndromes becomes possible in a more rigorous manner than before.

Keywords Behavioural consistency · Behavioural syndrome · Behavioural type · Personality · Syndrome deviation $\cdot$ Temperament

Main concepts in behavioural syndrome research

What is behavioural syndrome?

While correlations between different behaviours were described already a good while ago (Huntingford 1976), ethologists and behavioural ecologists continued to study behavioural traits (e.g. aggression, foraging) and ecological contexts (e.g. mating vs. non-mating seasons) in isolation from each other assuming case-by-case individual optimisation. However, the interest has recently turned towards analysing behavioural traits across different contexts (e.g. aggression towards competitors and own offspring) or analysing a suite of behavioural traits (e.g. aggression towards conspecifics and risk-taking in the presence of predators) together. When correlations across contexts and/ or behavioural traits exist, the resulting phenomenon bears a close resemblance to human personality (Gosling 2001). Such correlations are often referred to as "behavioural 
syndromes', ‘temperament' or 'animal personality' (for reviews on the concept see: Gosling 2001; Sih et al. 2004a, b; Bell 2007; Réale et al. 2007; Sih and Bell 2008). However, in our view, these terms are not to be used as synonyms. In this paper, we use the term 'behavioural syndrome' when referring to correlated behaviours and consider only correlations between different behaviours or behaviours in different contexts under such a term. We use the term 'temperament' when referring to a hypothetical individual characteristic that is expressed in different biological contexts as different behaviours (i.e. temperament is expressed as the level of aggression in an intrasexual competition context, or as the level of risk-taking in a predation risk context, etc.). We use the term 'animal personality' to describe individual consistency in the same behaviour across time.

Another important concept is 'personality trait'. In a recent review, Réale et al. (2007) classified five main personality trait categories (viz. shyness-boldness, exploration-avoidance, activity, sociability and aggression) to be studied in behavioural syndrome research. This classification helps in preventing the false identification of behavioural syndromes based on seemingly different behavioural traits, which in reality are just different measures of the same personality trait (e.g. number of aggressive displays, number of attacks, time spent with intruder, etc. are all measures of aggression, and will correlate without forming a behavioural syndrome). Here, we refer to personality traits in general when behaviours can be performed along a continuum potentially reflecting temperament. Traits that cannot be displayed along such a temperament axis, like food choice, song characteristics or accuracy of ectotherm thermoregulation, for instance, are regarded as irrelevant for the behavioural syndrome concept, and thus we do not treat them as personality traits as defined above. A perfect rankcorrelation between personality traits is necessarily +1 (and not -1 ), as individuals along each trait can always be ranked along an analogous temperament continuum by inverting the scale for one trait if necessary. We note that behavioural traits that are not personality traits by our definition (see above), i.e. they are not potential manifestations of temperament, can also be tested in correlations, and thus the approach outlined in this paper can be adapted to them after negative correlations are transformed to positive.

Finally, it is very important to make a clear distinction between the terms 'behavioural syndrome' and 'behavioural type' when multiple personality traits are studied on the same individuals. Behavioural syndrome refers to a "correlation between rank-order differences between individuals" (Bell 2007; Fig. 1), whereas behavioural type is defined as "a particular configuration of behaviours" (Bell 2007; Fig. 1). From these definitions, two important

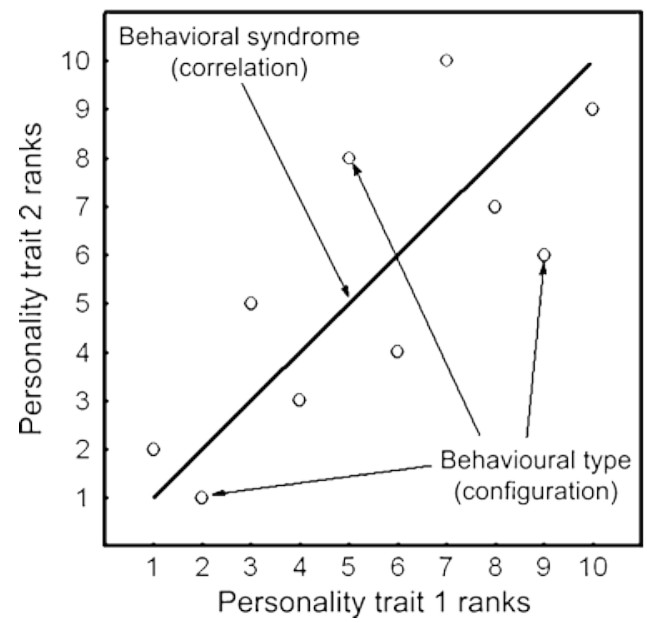

Fig. 1 Schematic representation of behavioural syndrome and behavioural type (modified after Bell 2007). Behavioural syndrome is the existence of a rank-order correlation between two personality traits (group-level trait) while behavioural type is the individual configuration of the personality traits (individual-level trait). Note that the line is shown only for illustrative purposes

realisations emerge. First, the presence or absence of behavioural syndromes can only be studied with reference to a group of individuals (usually population). Second, even when an individual is described using several personality traits, one cannot speak about behavioural syndromes, only about behavioural types (e.g. Herczeg et al. 2009; David et al. 2011).

Origins of behavioural syndromes: adaptive vs. constraint hypotheses and their limited testability

The most challenging evolutionary question associated with behavioural syndromes is the origin of the nonindependence of seemingly different personality traits like aggression and boldness towards predators. Two main hypotheses-known as the constraint and adaptive hypotheses-have been evoked to explain concerted evolution of personality traits (Bell 2005). The constraint hypothesis is based on the assumption that a shared proximate link, e.g. the same hormone affecting different behaviours (Ketterson and Nolan 1999), or genetic correlations (van Oers et al. 2005) between personality traits, are responsible for the behavioural syndrome. In contrast, the adaptive hypothesis states that correlations between personality traits emerge only when the correlation itself is adaptive (Bell 2005). In other words, according to the constraint hypothesis, behavioural syndromes constrain evolution, while according to the adaptive hypothesis, behavioural syndromes emerge as adaptive responses to selection favouring particular syndromes (for more details, see, e.g. Carere et al 2010, Dingemanse and Wolf 2010, Wolf and Weissing 2010). 
There are only a limited number of studies available where the results were (or can be) used to test the evolutionary hypotheses behind behavioural syndromes (Table 1). Furthermore, most of these studies suffer from logistic constraints, like low number of studied populations or the use of wildcaught individuals, making solid evolutionary inference challenging. The general problem with using wild-caught individuals in evolutionary studies is recognised (e.g. Merilä 2009). Furthermore, it has been shown how environmentally induced phenotypic plasticity is involved in the emergence of behavioural syndromes (Bell and Sih 2007). The single study to date that actually tested the correlation between environmental variation and the presence/absence of behavioural syndromes statistically (using wild-caught individuals) supported the adaptive hypothesis (Dingemanse et al. 2007). Despite this limited support for the adaptive nature of behavioural correlations, different explanations for the adaptive basis of behavioural syndromes have already been advanced (e.g. Stamps 2007, Wolf et al. 2007).

Most of the studies that investigated the evolutionary determinants of behavioural syndromes relied on a comparison of different populations of the same species experiencing different selection regimes (Table 1). Therefore, the basis for comparison is the presence/absence of the syndrome within a population, which is an all-or-nothing categorisation based on the significance of behavioural correlations observed within a population. In this way, between-individual variation in contributing to the behavioural syndromes within a population is disregarded, and selection acting on individuals remains unexplored. More- over, the comparison of populations necessarily suffers from low sample sizes, which are limited to the number of populations being compared, making generalisations difficult.

Perhaps the most significant problem in evolutionary ecology of behavioural syndromes is rooted in the definition of behavioural syndrome and how it can be measured. A behavioural syndrome is a presence/absence trait at the group level (Fig. 1), yet individual level estimates are needed both for quantitative genetic and fitness studies. Thus far, researchers have focused on analysing the quantitative genetics or fitness consequences of personality traits separately, without considering behavioural syndromes (e.g. Drent et al. 2003; Dingemanse et al. 2004, 2009; van Oers et al. 2004; Smith and Blumstein 2008). These results are very important for understanding the evolution of behaviour/ animal personality but do not directly help us in understanding the existence and evolution of behavioural syndromes.

The concept of syndrome deviation: individual-specific inconsistency in the performance of different behaviours

In a recent review, Dingemanse et al. (2010) proposed an exciting method (behavioural reaction norm approach) to study animal personality based on repeated measures of a personality trait in the same individuals along environmental gradients. This method allows us to uncouple animal personality and behavioural plasticity and to study their interaction (consistent individual differences in plasticity). By partitioning the variance in the same behavioural trait
Table 1 Studies where the presence/absence of behavioural syndromes was compared between populations. 'Conclusion' refers to conclusions regarding the competing evolutionary hypotheses behind behavioural syndromes. The question marks in the 'Conclusion' column denote cases where such conclusions cannot be drawn

\begin{tabular}{|c|c|c|c|c|c|c|}
\hline Study species & Study objects & $\mathrm{N}$ & Personality traits & Results & Conclusion & Study \\
\hline $\begin{array}{l}\text { Agelenopsis } \\
\text { aperta }\end{array}$ & $\begin{array}{l}\text { Wild-caught/F1 } \\
\text { common garden }\end{array}$ & 2 & Aggression, boldness & $\begin{array}{l}\text { Behavioural syndromes in } \\
\text { both populations }\end{array}$ & ? & $\begin{array}{l}\text { Riechert and Hedrick } \\
\text { (1993) }\end{array}$ \\
\hline $\begin{array}{l}\text { Gasterosteus } \\
\text { aculeatus }\end{array}$ & $\begin{array}{l}\text { F1 common } \\
\text { garden }\end{array}$ & 2 & $\begin{array}{l}\text { Activity, aggression, } \\
\text { boldness }\end{array}$ & $\begin{array}{l}\text { Behavioural syndromes in the } \\
\text { high but not in the low } \\
\text { predation population }\end{array}$ & $\begin{array}{l}\text { Rejecting the } \\
\text { constraint } \\
\text { hypothesis }\end{array}$ & Bell and Stamps (2004) \\
\hline G. aculeatus & Wild-caught & 2 & $\begin{array}{l}\text { Activity, aggression, } \\
\text { boldness }\end{array}$ & $\begin{array}{l}\text { Behavioural syndromes in the } \\
\text { high but not in the low } \\
\text { predation population }\end{array}$ & $\begin{array}{l}\text { Rejecting the } \\
\text { constraint } \\
\text { hypothesis }\end{array}$ & Bell (2005) \\
\hline G. aculeatus & Wild-caught & 12 & $\begin{array}{l}\text { Activity, aggressiveness, } \\
\text { exploration }\end{array}$ & $\begin{array}{l}\text { Behavioural syndromes in six } \\
\text { high but not in six low } \\
\text { predation populations }\end{array}$ & $\begin{array}{l}\text { Supporting the } \\
\text { adaptive hypothesis }\end{array}$ & $\begin{array}{l}\text { Dingemanse et al. } \\
\text { (2007) }\end{array}$ \\
\hline G. aculeatus & Wild-caught & 8 & $\begin{array}{l}\text { Activity, boldness, } \\
\text { exploration, learning }\end{array}$ & $\begin{array}{l}\text { Negligible evidence for } \\
\text { behavioural syndromes }\end{array}$ & ? & Brydges et al. (2008) \\
\hline $\begin{array}{l}\text { Pungitius } \\
\text { pungitius }\end{array}$ & $\begin{array}{l}\text { F1 common } \\
\text { garden }\end{array}$ & 4 & $\begin{array}{l}\text { Aggression, boldness, } \\
\text { feeding activity }\end{array}$ & $\begin{array}{l}\text { Negligible evidence for } \\
\text { behavioural syndromes }\end{array}$ & ? & Herczeg et al. (2009) \\
\hline $\begin{array}{l}\text { Anelosimus } \\
\text { studiosus }\end{array}$ & Wild-caught & 18 & $\begin{array}{l}\text { Attacking prey, } \\
\text { exploration/boldness, } \\
\text { social tendency }\end{array}$ & $\begin{array}{l}\text { Behavioural syndromes in all } \\
\text { populations }\end{array}$ & $?$ & Pruitt et al. (2010) \\
\hline
\end{tabular}

$\mathrm{N}$ number of populations 
across different contexts and different individuals based on Dingemanse et al. (2010), one can assess the withinindividual consistency of each behavioural trait along an environmental gradient. However, this approach does not deal with individual consistency across different behaviours. Accordingly, using Dingemanse et al.'s (2010) methodology, the evolution of animal personality (consistency in a single personality trait) can be studied rigorously, but it is not able to answer evolutionary questions about behavioural syndromes (the correlation between personality traits). In this paper, we provide a new method that allows us to analyse behavioural syndromes at the individual level and consequently study the evolution of behavioural syndromes in detail, and demonstrate this approach through an example analysis from a wild bird.

The correlation between behaviours, if it exists at all, is never perfect. Most studies have detected correlations with the strength of intermediate magnitude (median effect size across 115 estimates $r=0.22$; Dochtermann 2011). Even if a behavioural correlation is significant, the relatively weak correlations may imply that the assessment of behaviours is loaded with considerable noise. A recent meta-analysis of the repeatability of behaviour found, depending on the method used for calculation, a repeatability of $0.37-0.47$ on average (Bell et al. 2009). Statistical approaches are available that can account for the low repeatability of traits (Adolph and Hardin 2007), but the application of such correction still reveals moderate effect sizes for the relationships between behaviours $\left(r_{\text {corrected }}=0.31\right.$, when using the most conservative repeatability estimate and the mean of the number of measures per individual -0.37 and 4.41, respectively, from Bell et al. 2009). Therefore, the modest correlation between behaviours is only partially caused by the low repeatability of traits. The vast majority of the unexplained variance can be attributed to individuals that apparently do not follow the population-level behavioural syndromes (i.e. their behaviours are not predictable from each other). Therefore, the observed correlation between personality traits within a population is the net effect of individuals that show behaviours at different consistency: if most individuals display different personality traits with similar temperament (i.e. they are predictable), a syndrome is detected, but no syndrome can be revealed if many individuals are ranked unpredictably along different personality traits.

Using the concept of syndrome deviation-by quantifying the individual deviation from ( contribution to) the hypothetical perfect correlation-generates testable predictions at the individual level. The benefits of this concept are obvious. If population-level behavioural syndromes evolve by means of natural selection, three requirements must be met at the individual level (unless selection has already exhausted genetic variation): (1) There should be consistent variation in how much the individuals behave as predicted by the syndrome, and this individual attribute has to be (2) heritable and (3) linked to fitness. Hence, to understand the evolution of behavioural syndromes, the quantitative genetic parameters and the fitness of the individual contribution to the syndrome should be quantified to reveal its evolvability and to understand the selective forces resulting in the emergence of behavioural syndromes at the population level.

Importantly, syndrome deviation can be meaningful not only in cases when the correlation between behaviours is strong and significant but also when the correlation is weak and non-significant, and thus the behaviours (personality traits) do not form a syndrome. In the concept of syndrome deviation, the lack of a syndrome would imply that individuals that behave inconsistently constitute the major part of the population, while consistent individuals represent the minority. This is a biologically plausible situation, and if it indicates the selective advantage of deviant behaviours, it offers a potential explanation as to why under some circumstances we do not detect syndromes. Inconsistent behaviour may be advantageous when the environment changes in an unpredictable manner, since individuals with high behavioural plasticity or an inconsistent behavioural type may be better able to adapt to these changes. Moreover, when several environmental factors shape different behaviours in different parts of life, those individuals that can vary their behaviours independently from each other will be more likely to possess the most adaptive behavioural configuration.

How can we measure individual consistency across behaviours?

A variable is needed that describes how well a certain individual fits to the behavioural syndrome. Note that, strictly speaking, a behavioural syndrome is simply the existence of a rank-order correlation between two behaviours without considering the actual scale of behaviours, and that a 'perfect' rank-correlation (i.e. when the ranks are fully consistent) could only be +1 (Figs. 1 and 2). To understand why behavioural syndromes evolve, one must put aside whether a given individual is shy and peaceful or bold and aggressive (i.e. the behavioural type sensu Bell 2007 is irrelevant). The question is how well an individual fits to the hypothetical perfect syndrome (when the correlation between behaviours is $r=1$ ), as the relationship between the personality traits (more specifically the rank of individuals across different personality traits), and not the personality traits themselves, is supposed to be adaptive. The deviation of a given individual's behaviour from the one predicted by the hypothetical perfect syndrome (for visualisation, see Fig. 2) could be such a variable. Spearman's rank correlation coefficient, which is generally used 


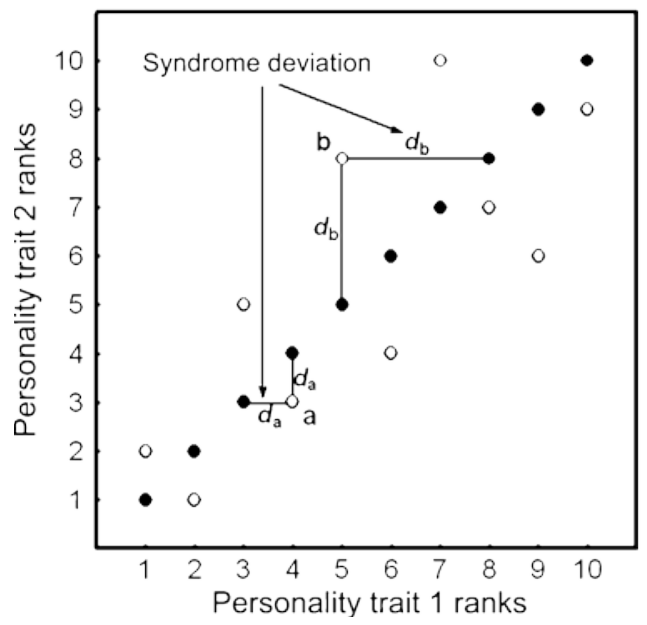

Fig. 2 Schematic representation of syndrome deviation, a new method of analysing behavioural syndromes at the individual level. Syndrome deviation describes how far an individual falls from the estimated perfect syndrome within the given population. Filled circles denote individuals from a perfect correlation, open circles denote individuals with an imperfect but still strong $\left(r_{s}=0.75 ; \mathrm{P}<0.05\right)$ correlation. Syndrome deviation is the absolute value of the individual deviation (d) from the perfect correlation, here shown for individuals ' $a$ ' and 'b'

for testing for the presence/absence of behavioural syndromes (e.g. Bell 2007), can be calculated as follows:

$r_{s}=\frac{6^{P} d_{1}^{2}}{n \succsim n^{2}-1 p}$

where $r_{s}$ is the coefficient, $d_{i}$ is the rank difference in a certain individual and $\mathrm{n}$ is the sample size (number of individuals measured). We note that, statistically, rankorder correlations can vary between +1 or -1 . However, correlations between personality traits are biologically meaningful on a scale that runs from 0 to +1 , assuming that personality traits are manifestations of the same temperament in different contexts (as personality traits are continuous measures, they can be simply inverted if needed). Hence, the absolute value of $d_{i}$ describes the individual deviation from the expectation $\left(d_{i}=0\right)$ under the hypothetical perfect correlation:

$$
S D_{i} 1 / 4 \mathrm{jd}_{\mathrm{i}} \mathrm{j}
$$

We coin this variable as 'syndrome deviation' (SYD), because it describes how far an individual falls from the hypothetical perfect syndrome within the given population (Fig. 2), or in other words, SYD is inversely related to how well an individual's ranks along different behaviours are predictable from each other. As the possible value of $d_{i}$ depends on the number of individuals tested (i.e. a larger number of individuals allows for larger deviations), similar sample sizes are recommended for studying multiple populations or measuring more than two personality traits.
When sample size vary between populations or differ across traits due to, for example, missing values, problems can arise because it alters the range of ranks. For instance, an individual that is consistently ranked last in all behaviours would receive different SYD scores if the ranks span different values for different behaviours. Therefore, when gathering similar sample sizes is impossible, $d_{i}$ should be corrected for $d_{\max }(=n-1)$ of the given population:

$\operatorname{relSYD} D_{i} 1 / 4 \frac{j d_{i} j}{n-1}$

However, because relative SYD (relSYD) (Eq. 3) is a ratio, large differences in sample size should be avoided (note that for the sake of simplicity, we use SYD for both simple SYD and relSYD hereafter when referring to syndrome deviation in a general sense). The obtained values can later be used for different comparisons within or between populations (e.g. between populations differing in predation pressure, between individuals with different fitness, etc.) or for calculating quantitative genetic parameters. The repeatability of SYD might also be calculated by repeated measures of the personality traits.

An extension for behavioural syndromes formed by more than two personality traits

Assuming that temperament is expressed in all personality traits simultaneously, a behavioural syndrome might include more than two personality traits. Accordingly, it might be interesting to calculate SYD across more than two personality traits.

The most intuitive approach would be to calculate SYD for all possible pair-wise combinations of behaviours and then to combine paired estimates across the whole sample of traits. Again, an important criterion to be met is that the derived SYD estimates should be comparable across different pairs of traits (i.e. sample size should be similar). If this criterion is violated, calculated SYD scores should be standardised by using Eq. 3 .

If paired estimates of SYD are available, it becomes possible to calculate the within-individual variation of SYD across pairs of traits. Such an estimate is of high importance because it provides information about the degree to which an individual deviates from the syndrome in general. If SYD is an individual-specific context-independent character that is heritable and subject to selection, it should be seen in every combination of personality traits considered. Such a consistent deviation should result in SYD estimates that are repeatable within individuals and across different pairs of traits. If there is evidence for repeatability (e.g. SYDs correlate across different pairs of personality traits), different sets of SYDs can be averaged, and low values will 
then subsequently indicate generally high consistency across all behaviours, while high values will represent individuals that systematically deviate from the hypothetical perfect syndrome. However, we can also imagine that selection variously favours different pairs of personality traits to form a syndrome (e.g. aggression and risk taking might coevolve due to predation pressure, while activity and exploration might vary non-independently due to a hormonal constraint). In this case, different SYD levels across pairs of traits might become adaptive for an individual, which would not require SYD to be repeatable (i.e. SYDs do not correlate across all pairs of personality traits), and in fact, would render overall SYD estimates calculated across all traits meaningless.

However, if such an overall individual-specific consistency index makes sense because the syndrome is expected to be multi-dimensional, one can calculate the consistency index across more than two behaviours without calculating paired estimates. Accordingly, we first need to adjust the scale of ranks if available sample sizes vary across traits and then estimate the variability in these standardised ranks. This approach is based on the expectation that individuals behaving as the syndrome predicts should be ranked similarly across different behaviours, while deviating individuals should receive variable ranks. In this case, the ranks (without ties), not their paired difference, should be brought into a common range (e.g. to 100 , or to the maximum of ranks in the behaviour with largest sample size). Such rank standardisation can be achieved by the following formula:

$y_{i} 1 / 4 a x_{i} p b$ ð4p

where $y_{i}$ is the standardised rank in the common scale, $x_{i}$ is the original rank,

a $1 / 4 \frac{R_{\max }=2}{x_{\max }-x_{\text {med }}}$

and

b $1 / 4 \frac{R_{\max }}{2}-a x_{\text {med }}$

with $R_{\max }$ standing for the highest rank in the common range (thus $R_{\max } / 2$ is the median rank in the common scale), $x_{\max }$ for the highest rank in the original scale and $\mathrm{X}_{\mathrm{med}}$ is the median rank in the original scale. Note that this standardisation also forces ranges to scatter around the same median $\left(R_{\max } / 2\right)$. Using standardised and centred ranks avoids the potential problems arising from dissimilar distributions of the original variables. If the common rank scale varies from 1 to 100 , the numerical value for an individual can be interpreted as the propor- tional rank occupied in the spectrum of the given personality trait within the whole population. The overall consistency can be described by a measure of variability of the standardised ranks across traits and within the individual (e.g. the standard deviation of ranks within an individual is a good multidimensional SYD estimate). Individuals that follow the syndrome will consistently have similar ranks across different behaviours and thus will demonstrate little variation, while conspecifics that behave inconsistently will show high variation in the standardised rank.

Again, the calculation of the SYD index across several behavioural traits relies on the assumption that all individuals are ranked similarly along all behaviours, i.e. from less active to the most active or from shy to bold, etc. The combination of all behaviours into a single consistency index does not allow the calculation of within-individual repeatability, but personality traits can be grouped into distinct biologically meaningful categories (e.g. based on contextual overlap), for which SYD indices are obtainable separately. Therefore, repeatabilities can be calculated across different contexts (e.g. between foraging and breeding sites, between captive and natural circumstances) or across different periods (e.g. between egg-laying and chick-feeding phases, between young and old ages), if multiple behavioural data are available from the same individual.

Above, we have derived two approaches to calculate SYD in cases when more than two personality traits are considered. The first method relies on a pair-wise comparison of ranked traits and the subsequent averaging of the paired difference between ranks. The second way is to standardise individual ranks along each trait considered and then to estimate the variance in these ranks. We suggest choosing between the two approaches depending on the biological question at hand. If the interest is to work with the syndrome concept in a pair-wise fashion (e.g. one expects different evolutionary background in the different combination of traits) or the repeatability of SYD within individuals is in focus, the first method may be the preferable option. On the other hand, if there is any reason to assume that the syndrome is composed of several personality traits, or repeatability of SYD across different contexts needs to be estimated, the second approach could be followed. In fact, our method allows us to test for the existence of multidimensional syndromes by calculating the repeatability of SYD across pairs of behavioural traits. If observed behaviours are the manifestation of the same temperament (or any other individual characteristics like parasite infection, etc.), individual deviations from the perfect correlation should be consistent, thus SYD should be consistent. However, if different mechanisms mediate the correlations be- 
tween different pairs of behaviours, one would not expect SYD to be repeatable across these pairs.

An example: syndrome deviation in collared flycatchers (Ficedula albicollis)

Data for this example originate from Garamszegi et al.'s (2008; 2009) collared flycatcher studies, from which we selected the following variables: exploration (exploration of a breeding environment that was altered with a novel object), risk-taking during aggression (the distance at which an individual involved in a territorial conflict flees from an approaching human), risk-taking during singing (song postexposure, i.e. the height of birds at singing relative to the surrounding vegetation) and aggression (latency to attack a territorial intruder). If necessary, variables were multiplied by -1 to obtain ranks that vary analogically. Ranks are
Fig. 3 Examples for behavioural syndromes and syndrome deviations. Each figure shows the pair-wise comparisons of individual ranks among four behavioural traits measured in collared flycatchers (Garamszegi et al. 2008, 2009). Lines ( $x=y)$ represent the expected perfect correlations according to the syndrome concept. The filled circle denotes one individual in the different pair-wise comparisons. For more details, see text
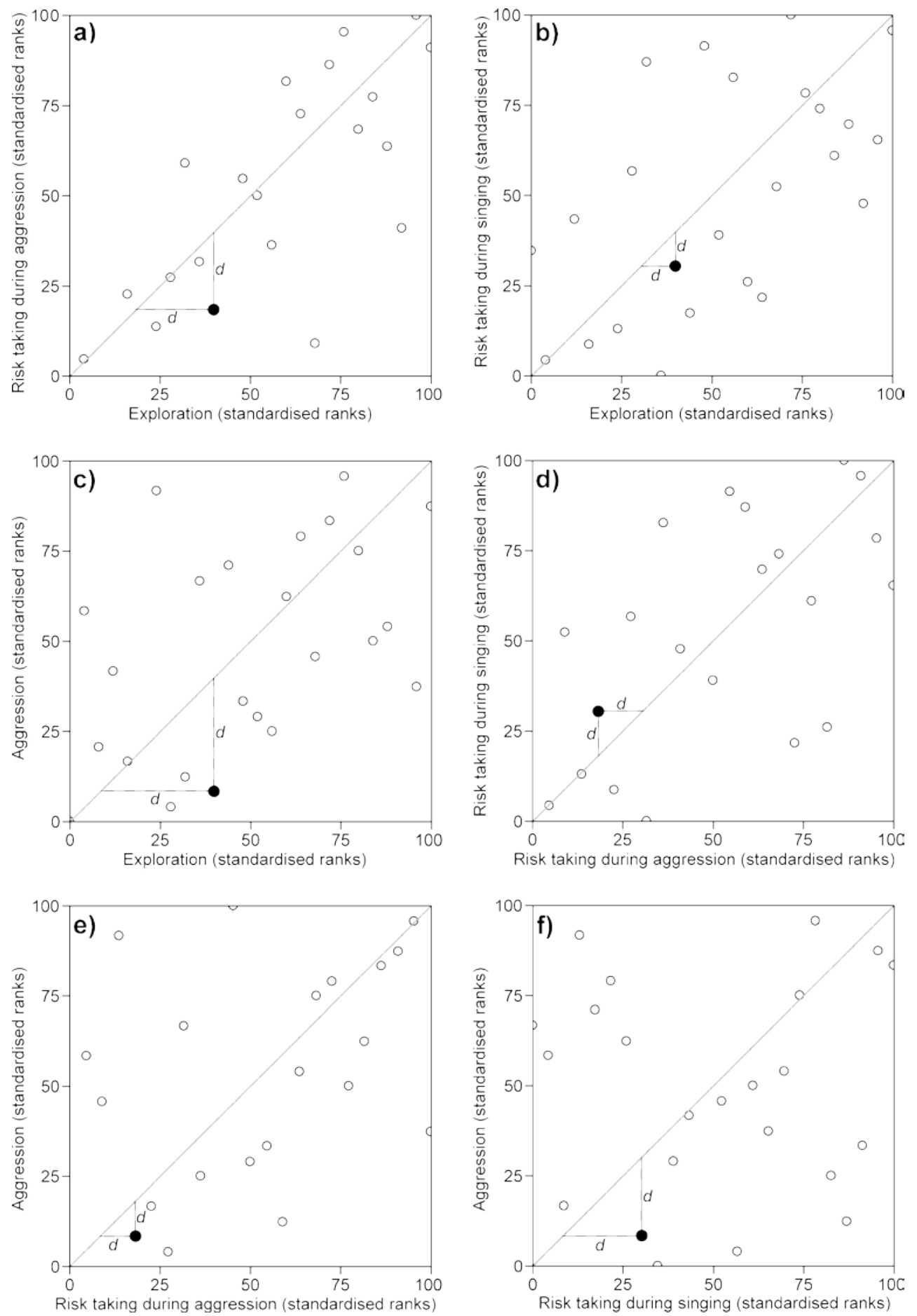
brought into a common range that varies from 1 to 100 by using the standardisation formulas 4-6. Hence, a standardised rank of 0 indicates the least 'active', while 100 the most 'active' individual in the given context.

Syndrome deviation (d in Fig. 3) from this expectation can be calculated as the absolute value of the pair-wise difference in the standardised ranks (see Formulas 1-3), which is graphically illustrated for one individual (black dots in Fig. 3). The correlations between the standardised ranks are: exploration and risk-taking during aggression, $r_{s}=0.757, N=22, P<0.001$ (Fig. 3a), exploration and risktaking during singing, $\mathrm{r}_{\mathrm{s}}=0.557, \mathrm{~N}=24, \mathrm{P}=0.005$ (Fig. $3 \mathrm{~b}$ ), exploration and aggression, $r_{s}=0.487, N=24, P=0.016$ (Fig. 3c), risk-taking during aggression and risk-taking during singing, $r_{s}=0.561, N=22, P=0.007$ (Fig. 3d), risktaking during aggression and aggression, $r_{s}=0.348, N=22$, $\mathrm{P}=0.112$ (Fig. 3e) and risk-taking during singing and aggression, $r_{s}=0.025, N=23, P=0.911$ (Fig. 3f).

There was evidence that the calculated deviation indices vary systematically within individuals implying that SYD is an individual-specific trait $\left(F_{23,111}=2.082, P=0.006\right)$. However, the repeatability of SYD as calculated across the pairwise comparisons (Fig. 3a-f) was modest $(R=0.163$, 95\% $\left.\mathrm{CI}_{\text {lower }}=0.018,95 \% \mathrm{CI}_{\text {upper }}=0.269\right)$ which suggests that not all personality traits are involved in the syndrome (this was also prevalent from the correlations of the standardised ranks). Given the consistent within-individual variation of SYD, it can be averaged across the six comparisons. Such a mean estimate is a good predictor of the withinindividual variation of the standardised ranks across the four personality traits (the correlation between the estimates of SYD based on our two different approaches: $r=$ $0.981, \mathrm{~N}=26, \mathrm{P}<0.001$ ). Therefore, estimating individualspecific estimates of SYD across the considered traits makes biological sense, and it would be straightforward to relate it to measures of fitness, which we plan to study in the future.

\section{Conclusions}

Syndrome deviation indices are a marked step forward because they will finally allow for the assessment of the individual variation behind the population-level behavioural syndromes. Thus, the main steps necessary in understanding the evolution of any trait, namely estimating its heritability and linking it to fitness, will be possible for behavioural syndromes using standard methodology. For instance, quantitative genetic parameters (additive/nonadditive genetic components, maternal effects, environmental effects, etc.) of SYD variation could be assessed either utilising special breeding designs in the lab, or long-term (multigenerational) datasets from natural populations. Fur- thermore, the fitness consequences of consistency/inconsistency in a suite of behaviours can be easily studied both in nature and in laboratory experiments by revealing the relationship between SYD and survival/reproductive success under various environmental conditions. Syndrome deviation indices will also allow the use of other approaches, like $\mathrm{Q}_{\mathrm{ST}}-\mathrm{F}_{\mathrm{ST}}$ comparisons to disentangle the roles of natural selection vs. drift (Merilä and Crnokrak 2001; Leinonen et al. 2008) in the emergence (or lack) of behavioural syndromes, or genome scans and QTL mapping (e.g. Schlötterer 2003; Erickson et al. 2004) to identify the genomic regions, and ultimately the genes coding for variation in the individual contribution to the syndromes. By adopting the concept of syndrome deviation, a new array of analyses on already existing data will become possible.

Acknowledgement Thanks are due to Juha Merilä for fruitful discussions that helped the hatching of ideas presented here and to Bob O'Hara for statistical advice. We are highly indebted to John Loehr for commenting on an earlier version of the paper and correcting the English. This work was supported by Academy of Finland (GH, ref. no. 128716), a Hungarian Scientific Research Fund (OTKA) (GH, ref. no. F6840), Spanish National Research Council (Consejo Superior de Investigaciones Científicas (CSIC)) (LZG, "Ramon y Cajal" research grant) and by the "Plan Nacional" program of the Spanish government (LZG, ref. no. CGL2009-10652 and CGL2009-09439).

\section{References}

Adolph SC, Hardin JS (2007) Estimating phenotypic correlations: correcting for bias due to intraindividual variability. Funct Ecol 21:178-184

Bell AM (2005) Behavioural differences between individuals and two populations of stickleback (Gasterosteus aculeatus). J Evol Biol 18:464-473

Bell AM (2007) Future directions in behavioural syndrome research. Proc R Soc B 274:755-761

Bell AM, Stamps JA (2004) Development of behavioural differences between individuals and populations of sticklebacks, Gasterosteus aculeatus. Anim Behav 68:1339-1348

Bell AM, Sih A (2007) Exposure to predation generates personality in three-spined sticklebacks (Gasterosteus aculeatus). Ecol Lett 10:828-834

Bell AM, Hankison SJ, Laskowski KL (2009) The repeatability of behaviour: a meta-analysis. Anim Behav 77:771-783

Brydges NM, Colegrave N, Heathcote RJP, Braithwaite VA (2008) Habitat stability and predation pressure affect temperament behaviours in populations of three-spined sticklebacks. J Anim Ecol 77:229-235

Carere C, Caramaschi D, Fawcett TW (2010) Covariation between personalities and individual differences in coping with stress: converging evidence and hypotheses. Curr Zool 56:728-740

David M, Auclair Y, Cézilly F (2011) Personality predicts social dominance in female zebra finches, Taeniopygia guttata, in a feeding context. Anim Behav 81:219-224

Dingemanse NJ, Wolf M (2010) Recent models for adaptive personality differences: a review. Phil Trans R Soc B 365:39473958 
Dingemanse NJ, Both C, Drent PJ, Tinbergen JM (2004) Fitness consequences of avian personalities in a fluctuating environment. Proc Roy Soc Lond B 271:847-852

Dingemanse NJ, Wright J, Kazem AJN, Thomas DK, Hickling R, Dawnay N (2007) Behavioural syndromes differ predictably between 12 populations of three-spined stickleback. J Anim Ecol 76:1128-1138

Dingemanse NJ, Van der Plas F, Wright J, Réale D, Schrama M, Roff DA, Van der Zee E, Barber I (2009) Individual experience and evolutionary history of predation affects expression of heritable variation in fish personality and morphology. Proc Roy Soc B 276:1285-1293

Dingemanse NJ, Kazem AJN, Réale D, Wright J (2010) Behavioural reaction norms: where animal personality meets individual plasticity. Trends Ecol Evol 25:81-89

Dochtermann NA (2011) Testing Cheverud's conjecture for behavioral correlations and behavioral syndromes. Evolution 65:1814-1820

Drent PJ, van Oers K, van Noordwijk AJ (2003) Realized heritability of personalities in the great tit (Parus major). Proc Roy Soc Lond B 270:45-51

Erickson DL, Fenster CB, Stenøien HK, Priec D (2004) Quantitative trait locus analyses and the study of evolutionary process. Mol Ecol 13:2505-2522

Garamszegi LZ, Eens M, Török J (2008) Birds reveal their personality when singing. PLoS One 3:e2647

Garamszegi LZ, Eens M, Török J (2009) Behavioural syndromes and trappability in free-living collared flycatchers, Ficedula albicollis. Anim Behav 77:803-812

Gosling SD (2001) From mice to men: what can we learn about personality from animal research. Psychol Bull 127:45-86

Herczeg G, Gonda A, Merilä J (2009) Predation mediated population divergence in complex behaviour of nine-spined stickleback (Pungitius pungitius). J Evol Biol 22:544-552

Huntingford FA (1976) The relationship between anti-predator behaviour and aggression among conspecifics of the three-spine stickleback. Anim Behav 24:245-260

Ketterson ED, Nolan V (1999) Adaptation, exaptation, and constraint: a hormonal perspective. Am Nat 154:S4-S25
Leinonen T, O’Hara RB, Cano JM, Merilä J (2008) Comparative studies of quantitative trait and neutral marker divergence: a meta-analysis. J Evol Biol 21:1-17

Merilä J (2009) It’s the genotype, stupid! J Anim Breed Genet 126:1-2

Merilä J, Crnokrak P (2001) Comparison of genetic differentiation at marker loci and quantitative traits. J Evol Biol 14:892-903

Pruitt JN, Riechert SE, Iturralde G, Vega M, Fitzpatrick BM, Avilés L (2010) Population differences in behaviour are explained by shared within-population trait correlations. J Evol Biol 23:748-756

Réale D, Reader SM, Sol D, McDougall PT, Dingemanse NJ (2007) Integrating animal temperament within ecology and evolution. Biol Rev 82:291-318

Riechert SE, Hedrick AV (1993) A test for correlations among fitnesslinked behavioural traits in the spider Agelenopsis aperta (Araneae, Agelenidae). Anim Behav 46:669-675

Schlötterer C (2003) Hitchhiking mapping-functional genomics from population genetic perspective. Trends Genet 19:32-38

Sih A, Bell AM (2008) Insights for behavioural ecology from behavioural syndromes. Adv Stud Behav 38:227-281

Sih A, Bell A, Johnson JC (2004a) Behavioural syndromes: an ecological and evolutionary overview. Trends Ecol Evol 19:372-378

Sih A, Bell AM, Johnson JC, Ziemba RE (2004b) Behavioral syndromes: an integrative overview. Q Rev Biol 79:241-277

Smith BR, Blumstein DT (2008) Fitness consequences of personality: a meta-analysis. Behav Ecol 19:448-455

Stamps JA (2007) Growth-mortality trade-offs and 'personality traits' in animals. Ecol Lett 10:355-363

van Oers K, Drent PJ, de Jong G, van Noordwijk AJ (2004) Additive and nonadditive genetic variation in avian personality traits. Heredity 93:496-503

van Oers K, de Jong G, van Noordwijk AJ, Kempenaers B, Drent PJ (2005) Contribution of genetics to the study of animal personalities: a review of case studies. Behaviour 142:1191-1212

Wolf M, Weissing F (2010) An explanatory framework for adaptive personality differences. Phil Trans R Soc B 365:3959-3968

Wolf M, van Doorn GS, Leimar O, Weissing FJ (2007) Life-history trade-offs favour the evolution of animal personalities. Nature 447:581-585 